\title{
Primary alveolar hypoventilation and XXXXY chromosopathy
}

\author{
D. Samolski1, A. Antón1, M. Mayos¹, M. Subirana², R. Güell1
}

ABSTRACT: Primary alveolar hypoventilation and $X X X X Y$ chromosopathy. D. Samolski, A. Antón, M. Mayos, M. Subirana, R. Güiell.

The association of primary alveolar hypoventilation (PAH) and chromosomic diseases has not been described previously. A 19 year-old man with Fraccaro's syndrome (XXXXY karyotype) was admitted to evaluate chronic hy- percapnic respiratory failure, pulmonary arterial hypertension and cor pulmonale. PAH was diagnosed. As effective treatment, such as non-invasive positive pressure ventilation (NIPPV), is available for this disorder we should intensify the search for $\mathrm{PAH}$ in patients with chromosome disease.

Monaldi Arch Chest Dis 2006; 65: 3, 169-171.

Keywords: Primary alveolar hypoventilation, chromosome diseases, noninvasive ventilation.

1 Pneumology Dept. Hospital de la Santa Creu i Sant Pau, Barcelona Spain.

2 Cardiology Dept. Hospital de la Santa Creu i Sant Pau, Barcelona Spain.

Correspondence: Daniel Samolski M.D., Pneumology Dept., Hospital de la Santa Creu i Sant Pau, Sant Antoni Ma Claret 167, 08025 Barcelona Spain; e-mail: dsamolski@hsp.santpau.es

\section{Introduction}

Primary alveolar hypoventilation $(\mathrm{PAH})$ is a rare clinical entity characterised by respiratory failure in the absence of pulmonary parenchyma or respiratory pump abnormalities. Diagnosis of PAH requires the presence of hypoventilation (hypercapnia) and the demonstration of normal respiratory function tests, especially during voluntary ventilation maneuvers. Functional disorders of the respiratory centre [1-3] are also a constant feature. Known diseases of the central nervous system must be ruled out to support this diagnosis [1-4]. The main objectives of the present study are to report a case of PAH associated to a chromosome disease (Fraccaro's syndrome), to describe clinical manifestations of this unusual syndrome, and to discuss the clinical implications of PAH in genetic diseases.

\section{Clinical Case}

We describe a 19 year-old man diagnosed with Fraccaro's syndrome in childhood (chromosome disease with XXXXY karyotype). He presented the typical phenotype characteristics of this entity: mental retardation, radioulnar synostosis, hypogonadotropic hypogonadism and congenital heart disease (persistent ductus arteriosus and interventricular communication, surgically resolved at the age of 3 months and 4 years, respectively). The patient remained cardiologically stable in spite of moderate pulmonary arterial hypertension manifested by mean pulmonary arterial pressure (mPAP) up to $50 \mathrm{mmHg}$. Over the last few years, the patient had suffered from progressive dyspnea, evolving from functional class II (NYHA) to III-IV, and also diurnal sleepiness. Physical examination showed cor pulmonale syndrome. Blood tests were normal. Chest $\mathrm{X}$ ray revealed only an increased cardio-thoracic index. A thoracic Computed Tomography $(\mathrm{CT})$ scan also demonstrated the presence of pulmonary hypertension signs without pulmonary thromboembolic disease or intravascular clots. Arterial blood gases demonstrated hypercapnic respiratory failure $\left(\mathrm{PaO}_{2} 48 \mathrm{mmHg} \mathrm{PaCO}_{2} 49 \mathrm{mmHg} \mathrm{pH}\right.$ 7.37). A transthoracic echocardiographic test showed severe dilation and dysfunction of right ventricle and $\mathrm{mPAP}$ of $70 \mathrm{mmHg}$. A polysomnographic recording performed immediately after these findings evidenced the presence of non-obstructive episodes of hypopnea and a constant decrease in arterial oxygen saturation $\left(\mathrm{SpO}_{2}\right)$ suggestive of hypoventilation (fig. 1). The patient's mental disorder ruled out the possibility of performing respiratory functional tests. However, he was able to complete a eucapnic hypoxia stimulus test. This test was evaluated using Rebuck and Campbell's method to assess the ventilatory response to hypoxia [5]. It demonstrated poor ventilatory response: $\boldsymbol{\Delta} \mathrm{MV} / \boldsymbol{\nabla} \mathrm{PaO}_{2} 0.13 \mathrm{~L} / \mathrm{min} / \mathrm{mmHg}, \boldsymbol{\Delta} \mathrm{MV} / \boldsymbol{\nabla} \mathrm{SpO}_{2}$ $0.13 \mathrm{~L} / \mathrm{min} / \%, \boldsymbol{\Delta} 01 / \nabla \mathrm{SpO}_{2} 0.02 \mathrm{cmH}_{2} \mathrm{O} / \%$. With the diagnosis of central hypoventilation syndrome associated with a chromosomic disease, which presents as respiratory failure and cor pulmonale, we decided to begin anti-coagulation treatment and non-invasive positive pressure ventilation (NIPPV). We used a bi-level positive pressure ventilator (VPAP III, ResMed, Australia) with its own single limb circuit and a nasal mask (UltraMirage, ResMed, Australia). Using the common techniques for NIPPV adaptation, we reached an IPAP 


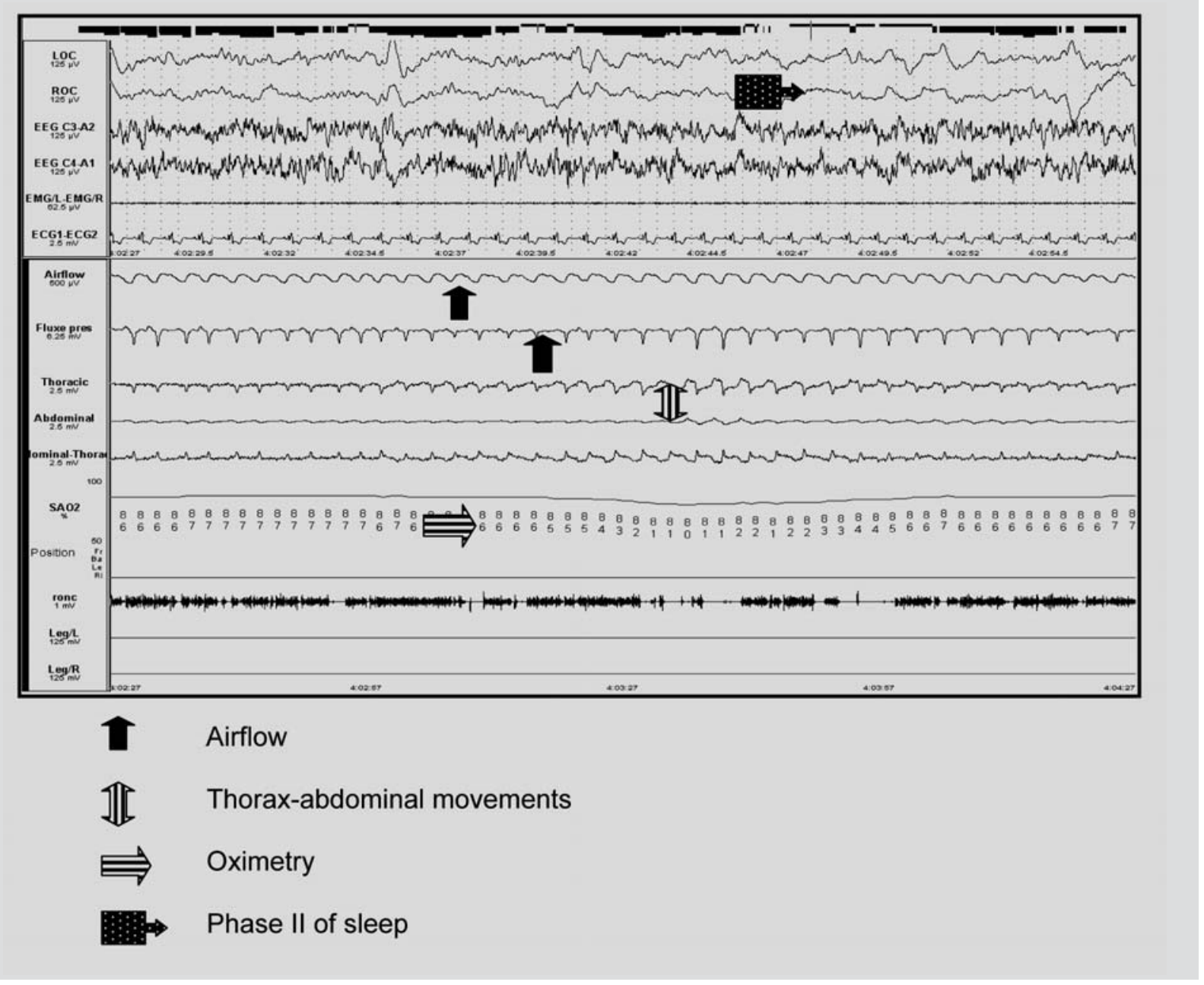

Figure 1. - Polysomnographic* recording showing constant decrease in $\mathrm{SpO}_{2}$ without obstructive respiratory disorders.

* Polysomnographic studies (Ultrason WS, Nicolet, Madison,Wisconsin) included the recording of electroencephalogram ( 2 unipolar leads), left and right electrooculogram and chin electromyogram for sleeping staging, electrocardiogram, oro-nasal airflow with thermistor and nasal cannula, rib cage and abdominal wall displacement measured by piezolelectric bands, oxyhemoglobin saturation and snoring by stenal notch. Sleep staging was manually interpreted in 30 -second periods according to standard criteria. Apnea was defined as an airflow cessation lasting > 10 seconds. Hypopnea was defined as an airway reduction lasting for > 10 seconds associated with a $3 \%$ decrease in saturation level and or an arousal. Arousals were scored according to the American Sleep Disorders Association recommendation [20]. $\mathrm{SpO}_{2}$ : arterial oxygen saturation measured by pulse oxymetry

of $16 \mathrm{cmH}_{2} \mathrm{O}$ and an EPAP of $6 \mathrm{cmH}_{2} \mathrm{O}$. Clinical and blood gas response to NIPPV was not as good as we expected. This was probably related to cognitive alterations, which affected treatment tolerance and compliance. A pulse-oxymetry evaluation and blood gas analysis performed prior to hospital discharge showed a small improvement in $\mathrm{SpO}_{2}$ and $\mathrm{PaO}_{2}$, associated with a slight decrease in $\mathrm{PaCO}_{2}$. Blood gas analyses continued to show oscillations in later follow-ups.

\section{Discussion}

Fraccaro's syndrome, named after the physician who first described it in 1960 [6], is a very rare chromosomic aneuploidy (XXXXY karyotype) with an incidence of about 1/ 85,000 live births [7-10]. It is characterised by mental retardation, typical facies, radioulnar synostosis, hypogonadotropic hypogonadism and congenital heart disease (mainly, persistent ductus arteriosus) in $20 \%$ of cases. Only 100 cases have been described to date and none so far have described the ventilatory disorders observed in our patient.

Morales et al. [11] described a potential relationship between chromosomic diseases and respiratory pathology, although they did not report the presence of a ventilatory control disorder. They demonstrated the existence of restrictive disease in patients with Klinefelter syndrome (XXY karyotype), expressed by reduced total lung capacity and normal $\mathrm{FEV}_{1} / \mathrm{FVC}$ ratio. Maximal inspiratory pressure was also normal and there was a reduced pulmonary compliance. They suggested that the reduction in pulmonary matrix elasticity, together with the aforementioned reduced compliance, could be due to testosterone deficiency. Against this idea is the lack of improvement in respiratory dysfunction with testosterone replacement therapy treatment [11]. In the present case, the patient had been receiving this hormone since the age of 13 .

To our knowledge, this is the first case describing PAH in a patient with a XXXXY chromosome disease. We have not found any reports in the 
literature regarding the relationship between chromosome disease and respiratory centre dysfunction. However, the relationship between those disorders and other components of the nervous system such as autonomic dysfunction is better known. Alterations of the sympathetic-parasympathetic balance may cause cardiovascular and/or respiratory dysregulations [3, 12-14]. This imbalance explains the appearance of cardiac arrhythmias, and decreased beat-to-beat variability during sleep, as well as the association of this entity with others which reflect autonomic dysfunction such as Hirschsprung's disease [15], pupil abnormalities, profuse perspiration and decrease in body temperature [3].

A report on PAH in monozygotic twins suggests that the PAH syndrome may have a genetic component [16]. In another study, a series of adult women with central congenital hypoventilation syndrome (CCHS) had children with this disease [15] supporting a genetic basis in CCHS. Knowing the association between CCHS and Hirschprung's disease (20\% of the patients), the genes involved could be those which codify for tyrosin kinase receptor, glia -derived neurotrophic factor, and endothelin receptor. The PHOX2B gene which codifies for a transcription factor to promote neuronal differentiation, is also been implicated in CCHS [14]. No one gene potentially related to this syndrome has been linked to the sex chromosome. There is consequently a lack of knowledge regarding whether the supernumerary X chromosome (as present in our patient) could play a role in the etiopathogenesis of central hypoventilation syndrome.

Hypoventilation and impaired cor pulmonale could be under-estimated in these patients, since part of their symptomatology is generally attributed to their cognitive deterioration and cardiac disorders. Presently available treatments, such as NIPPV, have proven useful in patients with hypoventilation disorders $[18,19]$. For this reason, diagnosis of central hypoventilation in patients with this type of chromosome disorder is of great importance, since early prescription of home mechanical ventilation may modify the natural course of these diseases.

\section{References}

1. Martin TJ, Sanders MH. Sleep-disordered breathing. State of the art review. Chronic alveolar hypoventilation: a review for the clinician. Sleep 1995; 18: 617-634.

2. Shneerson J. Disorders of automatic control of ventilation. In: Schneerson J, editor. Disorders of ventilation. Oxford: Blackwell Scientific Publications, 1998; p. 118-127.
3. ATS guidelines. Idiopathic congenital central hypoventilation syndrome: Diagnosis and management. Am J Respir Crit Care Med 1999; 160: 368-373.

4. McNicholas WT, Philipson EA. Central hypoventilation syndromes and hypercapnic central sleep apnea. In: WB Saunders, editor. Breathing disorders of sleep. Harcourt Publishers Limited. London, 2002; pp. 239-245.

5. Rebuck AS, Campbell EJM. A clinical method for assessing the ventilatory response to hypoxia. Am Rev Respir Dis 1974; 109: 345-350.

6. Fraccaro M, Kaijser K, Lindsten J. A child with 49 chromosomes. Lancet 1960; 2: 899-902.

7. Peet J, Weaver D, Vance GH. 49 XXXXY: a distinct phenotype. Three new cases and review. J Med Genet 1998; 35: 420-424.

8. Sheridan MK, Radlinski S. A case study of an adolescent male with XXXXY Klinefelter's syndrome. $J$ Aut Disorders 1988; 18 (3): 449-456.

9. Verotti A, Chiarelli F, Violante N, et al. La sindrome 49 XXXXY, dezcrizione di due casi clinici. Ped Med Chir 1986, 8: 575-8.

10. Curfs LMG, Schreppers-Tijdink A, Wiegers A, et al. The 49 XXXXY syndrome: clinical and psychological findings in five patients. Journal Mental Deficiency Research 1990, 34: 277-282.

11. Morales P, Furest I, Marco V, et al. Pathogenesis of the lung in restrictive defects of Klinefelter's Syndrome. Chest 1992; 102: 1550-52.

12. Woo MS, Woo MA. Heart rate variability in congenital central hypoventilation syndrome. Pediatr Res 1992; 31: 291.

13. Haddad GG, Mazza NM, Defendini R, et al. Congenital failure of automatic control of ventilation, gastrointestinal motility and heart rate. Medicine (Baltimore) 1978; 57: 517-526.

14. Weese-Mayer D, Berry-Kravis M. Genetics of congenital central hypoventilation syndrome. Am J Respir Crit Care Med 2004; 170: 16-21.

15. Hamilton J, Bodurtha JN. Congenital central hypoventilation syndrome and Hisrschprung's disease in half sibs. J Med Genet 1989; 26: 272-274.

16. Khalifa MM, Flavin MA, Wherrett BA. Congenital central hypoventilation syndrome in monozygotic twins. J Pediatr 1988; 113: 853-855.

17. Sritippayawan S, Hamutcu R, Kun SS, et al. Motherdaughter transmission of congenital central hypoventilation syndrome. Am J Respir Crit Care Med 2002; 166. 367-369.

18. Simonds AK, editor. Non invasive respiratory support: a practical handbook, $2^{\text {nd }}$ edition. London: Arnold publishers; 2001.

19. Leger P, Hill N. Nasal positive pressure ventilation in restrictive thoracic and central hypoventilation disorders. In: Hill NS, editor. Noninvasive positive pressure ventilation: principles and applications. Armonk, New York: Futura Publishing Company, Inc.; 2001: p. 121144.

20. American Academy of Sleep Medicine Task Force. Sleep-related breathing disorders in adults: recommendations for syndrome definition and measurement techniques in clinical research. Sleep 1999: 22; 667-89. 